\title{
Orthopaedic Timing in Polytrauma in a Second Level Emergency Hospital. An Overrated Problem?
}

\author{
L. Dei Giudici*, N. Giampaolini, A. Panfighi, M. Marinelli, R. Procaccini and A. Gigante
}

Clinical Orthopaedics, Department of Clinical and Molecular Science, School of Medicine, Università Politecnica delle Marche, Italy

\begin{abstract}
The main concern for orthopaedic treatment in polytrauma has always been the same for almost forty years, which also regards "where" and "when" to proceed; correct surgical timing and correct interpretation of the DCO concept are still being debated. In the last few years, several attempts have been made to classify patients based on their clinical presentation and by trying to figure out which vital parameters are able to predict the patient's outcome. This study evaluated all patients who presented with code red at the Emergency Department of our Hospital, a level II trauma center. For every patient, the following characteristics were noted: sex, age, day of hospitalization, orthopaedic trauma, time to surgery, presence of an associated surgical condition in the fields of general surgery, thoracic surgery, neurosurgery and vascular surgery, cardiac frequency, blood pressure, oxygen saturation, Glasgow Coma Scale and laboratory data. All patients included were divided into subgroups based on orthopaedic surgical timing. Two other subgroups were also identified and analyzed in detail: deceased and weekend traumas. A total of 208 patients were included. Our primary goal was to identify a correlation between the mortality and surgical timing of the orthopaedic procedures; our secondary goal was to recognize, if present, a statistically relevant association between historical, clinical and laboratory data, and mortality rate, defining any possible risk factor. A correlation between mortality and orthopaedic surgical timing was not found. Analyzing laboratory data revealed an interesting correlation between mortality and: blood pressure, platelet count, cardiac frequency, hematocrit, hemoglobin and age.
\end{abstract}

Keywords: Emergency, mortality, orthopaedic trauma, predisposing factors, polytrauma, surgical timing.

\section{INTRODUCTION}

Polytrauma defines the medical condition of a person who has been subjected to multiple traumatic injuries such as serious head, abdominal, thoracic, vascular injuries, and single or multiple fractures. It is often associated with motor vehicle accidents, or in general with high-energy impacts, and frequently represents a life-threatening condition; the concomitance of multiple organic and bone lesions can, in fact, destabilize a patient's hemodynamics due to, for instance, severe blood loss. Prompt and early care for those patients is therefore mandatory which requires a strict respect of clinical priorities in order to hemodynamically re-stabilize the patient and to have the best chances of saving his or her life. While for some kinds of injuries an emergency surgery is a "must", e.g., a spleen rupture, for others, and specifically for orthopaedic conditions, there is still an ongoing debate on their surgical timing. Moreover, no study has yet clearly stated what historical, clinical, instrumental or laboratory data are of good use for a Trauma Leader or the orthopaedic surgeon when it comes to decide whether a specific fracture needs to be treated as soon as possible, if the proposed treatment must be definitive, or whether there is the need to delay orthopaedic surgery.

*Address correspondence to this author at the Clinical Orthopaedics, Department of Clinical and Molecular Science, School of Medicine, Università Politecnica delle Marche, via Tronto, 10/A, 60126, Ancona, Italy; Tel: +39 328 5964024, +39 071 5963346; Fax: +39 071596 3341;

E-mail: lucadeigiudici@gmail.com
The principles for fracture management in a polytrauma patient, therefore, are still being debated in the scientific community. The main concern for an orthopaedic treatment in polytrauma has always been the same for almost forty years, which also regards "where" and "when" to proceed [1]. Historically, procedural protocols went from Kuntschers' recommendations in 1967 advising to procrastinate each and every orthopaedic surgery for some days [2-5], to the eighties trend of "early fixation" (ETC), promoting a definitive treatment as soon as possible [6-10]. Only during the '90s, after dedicated researches [11-14], the concept of damage control orthopaedics (DCO) started to be defined, which suggested temporary fracture stabilization for the most critical and unstable patients and allowing for the stabilization of the patient's hemodynamics as well.

The first practical application of this new therapeutic strategy was external fixation, while lately also closed reduction with internal fixation with an endomedullary nail has been taken into account for both primary and definitive care [8-12].

Correct surgical timing and correct interpretation of the DCO concept are still being debated [2, 3, 15, 16], and universally accepted guidelines are not yet available.

In the last few years, several attempts have been made to classify patients based on their clinical presentation [1-17], trying to figure out which vital parameters are able to predict the patient's outcome and, therefore, lead the surgeon to decide on proper management in terms of surgical timing and primary technique $[2,3,18-22]$. 
The purpose of the present study was double. The primary goal was to identify a possible correlation between the mortality and surgical timing of the orthopaedic procedures in patients who had been victims of polytrauma, admitted to our Hospital in a 3-year period. The secondary goal was to recognize whether there exists a statistically relevant association between historical, clinical and laboratory data, and mortality rate, defining any possible risk factor.

\section{MATERIALS AND METHODS}

This study evaluated all patients who presented with code red at the Emergency Department of our hospital, a level II trauma center with a catchment area of 1.5 million people in a time frame ranging from $1^{\text {st }}$ January 2008 to $31^{\text {st }}$ December 2010.

The inclusion criteria were: polytraumatic accident and the presence of at least one lesion involving the musculoskeletal system.

The only exclusion criterion was age below 14 as these young patients are always addressed to a specialized pediatric hospital of the city.

Data on patients were gathered by retrospectively reviewing the hospital's computer records. Triage Informatic Records (PS Web, Fly Technologie \& Servizi S.c.c.a.r.l., Perugia, Italy) were reviewed first, filtering records for code red only and extrapolating those presenting with an orthopaedic condition, e.g., a fracture, or for whom the examination by an orthopaedic surgeon was required. Out of this preliminary group, a second review was made of the Surgical Procedures Registry (Ormaweb, Dedalus Healthcare Systems Group s.p.a., Firenze, Italy) to divide surgical patients from non surgical ones. At this stage, any procedure performed in an emergency setting was noted; orthopaedic surgical timing was noted as well, even if definitive procedure for that particular condition was delayed. Lastly, a double check was performed by analyzing Radiological Imaging Database (Centricity Enterprise Web, GE Medical Systems Information Technologies, GE Healthcare, Little Chalfont, United Kingdom) for a specific patient since the time of her/his admittance at the emergency department onward in order to confirm her/his inclusion in either the surgical or non surgical patient group. Discharge records (SIO Web, Fly Technologie \& Servizi S.c.c.a.r.l., Perugia, Italy) were reviewed in order to identify those patients that had died.

In regards to the hospital's Privacy Policy, it must be noted that every reported system and record provided were sorted by an anonymous identification number assigned at the admittance to the hospital, and that this retrospective study has been approved by the Local Ethical Committee.

The final cohort was therefore widely assorted with a high variability of lesions, both orthopaedic specific and collateral.

For every patient, the following characteristics were noted: sex, age, day of hospitalization, orthopaedic trauma, time to surgery, presence of an associated surgical condition in the field of general surgery, thoracic surgery, neurosurgery and vascular surgery, cardiac frequency, blood pressure, oxygen saturation, Glasgow Coma Scale.

The following laboratory blood data were gathered from the computer system (Alchymia ${ }^{\circledR}$, S.C.S. Software \& Computer Systems, Fermo, Italy) at the time of admittance: leukocyte count, blood red cells, hemoglobin, hematocrit, platelet count. The monitoring of these parameters is part of the standard management in our hospital; registering and analyzing them for this paper was aimed at the identification of risk categories and variables, which are able to influence mortality. The last data assessed were mortality; the several degrees of disability suffered after recovery were not studied in this paper.

All patients included were divided into subgroups based on orthopaedic surgical timing, i.e., those who were treated: under 6 hours, between 6 and 12 hours, between 12 and 24 hours, between 24 and 48 hours, between 48 and 72 hours and after 72 hours; mortality was related to those intervals.

Two other subgroups were also identified and analyzed in detail: deceased and weekend traumas. The purpose of this division was to better respond to our study's secondary endpoint and to evaluate whether the anecdotal statement that weekend days have higher mortality is true or false.

Statistical analysis comprehended ranges, mean values, percentage values, standard deviations of all quantitative values; Fisher's Test was applied to identify possible and statistically significant associations between recorded data.

\section{RESULTS}

A total of 208 patients met the inclusion criteria and were included.

The study group consisted of 155 males $(74.52 \%)$ and 53 females $(25.48 \%)$, with a male to female ratio of 2.92 and a mean age of 43.25 ( $\min 14 y o, \max 84 y o$, SD 19.43); 120 patients $(57.69 \%)$ were admitted to the Emergency Department during week days, while 88 patients $(42.31 \%)$ were admitted during weekends.

All patients presented with at least one lesion involving the musculoskeletal system, as shown in Fig. (1). For a total of 163 patients $(78.37 \%)$, surgery was indicated; it was not indicated in 30 patients $(14.35 \%)$ and not possible due to exitus in 15 patients $(7.21 \%)$. Surgical timing was divided into subgroups, which is shown in Fig. (2); for 3 patients, timing was not recorded. The majority of the patients (115 patients, 55.29\%) were assessed with an ETC approach, while $48(23.08 \%)$ underwent a first surgery for DC.

As for associated surgery, distribution is provided in Fig. (3). In the general surgery group, 6 patients $(46.15 \%)$ presented with a condition that was to be treated by general surgeons as the only association to the orthopaedic condition; 3 cases (23.08\%) had also an associated condition that had to be treated by thoracic surgeons, and in 2 cases $(15.38 \%)$ there was an association with a neurosurgical or vascular surgical condition. Three people died in this subgroup, representing $1.44 \%$ of the total; for 2 of them, their orthopaedic condition was associated with both general and thoracic surgery conditions, while the remaining one 


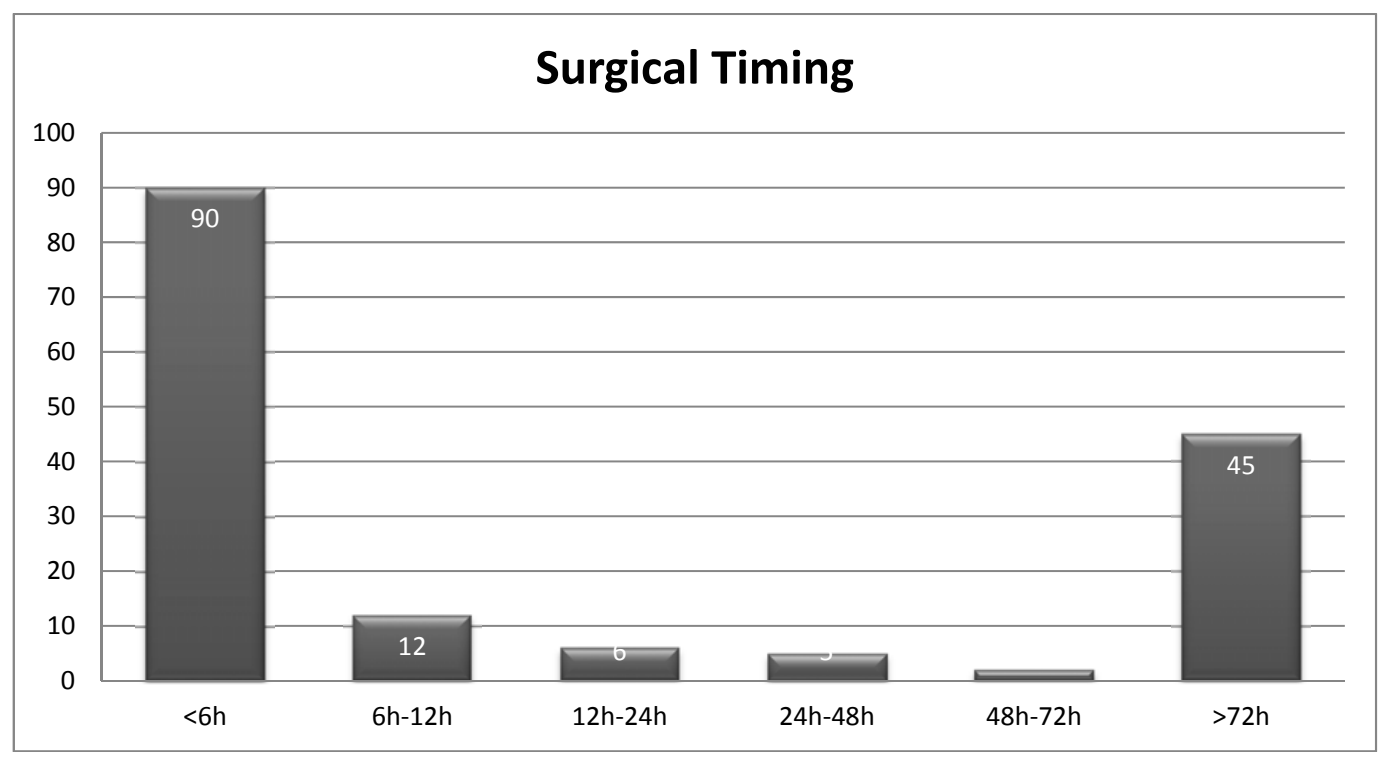

Fig. (1). Distribution of orthopaedic conditions presenting at the emergency department during the 3-year time span.

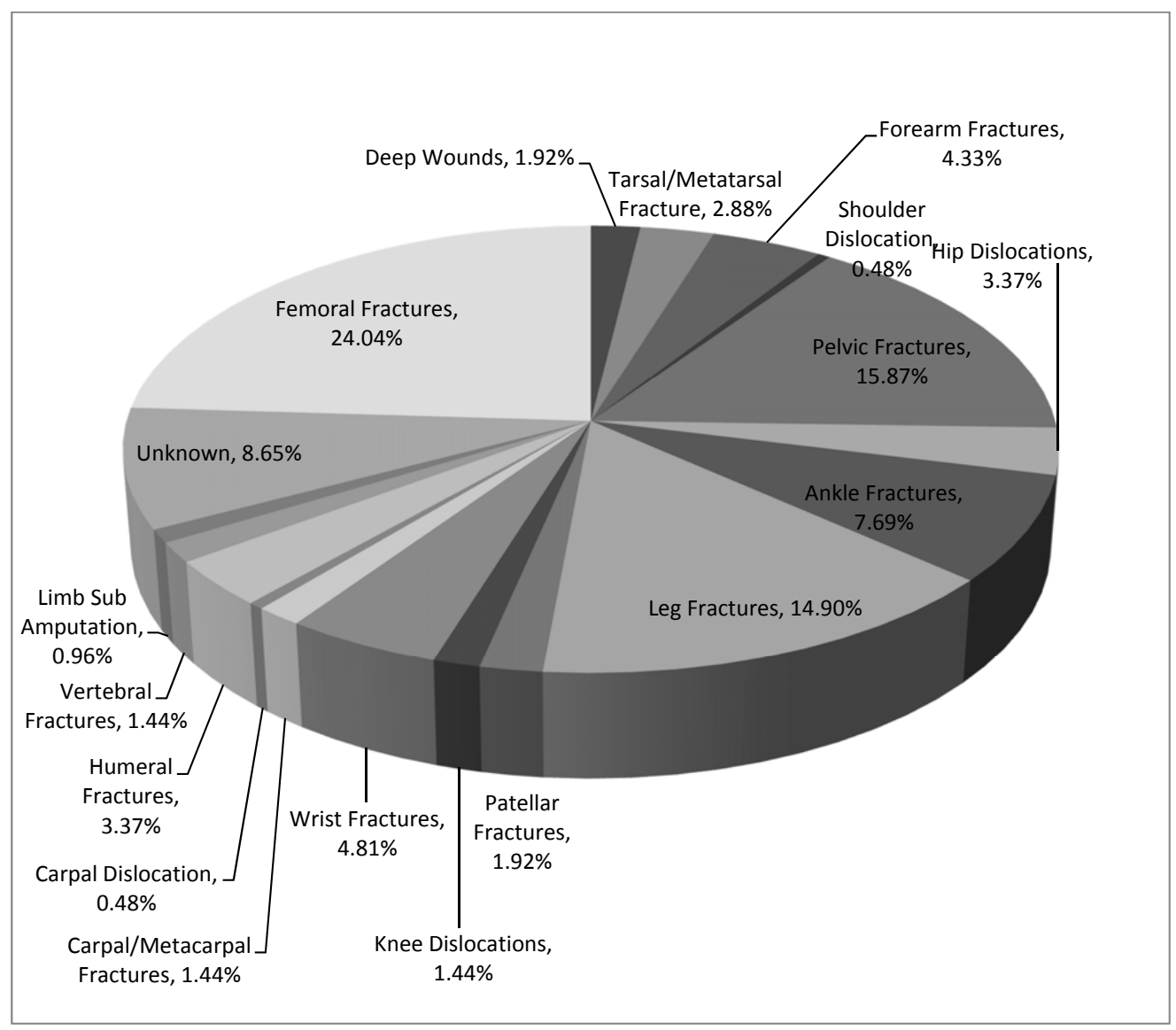

Fig. (2). Surgical timing; number of patients divided based on the time span passed from admittance to surgery.

patient had an associated condition with general surgery and vascular surgery conditions.

A thoracic condition that had to be treated surgically was never the only association with the orthopaedic condition. In 3 patients, there was also a condition that had to be treated by general surgeons, and in 1 of them, there was also a neurosurgical condition; two patients died $(0.96 \%$ of the total cohort), who presented with a condition associated with the orthopaedic condition, which had to be treated by both general and thoracic surgeons.

A neurosurgical condition was the only associated condition in $84.62 \%$ of the cases. Beside the orthopaedic condition, it was associated with a condition that had to be treated by general surgeons in $7.69 \%$ of the cases, while it 


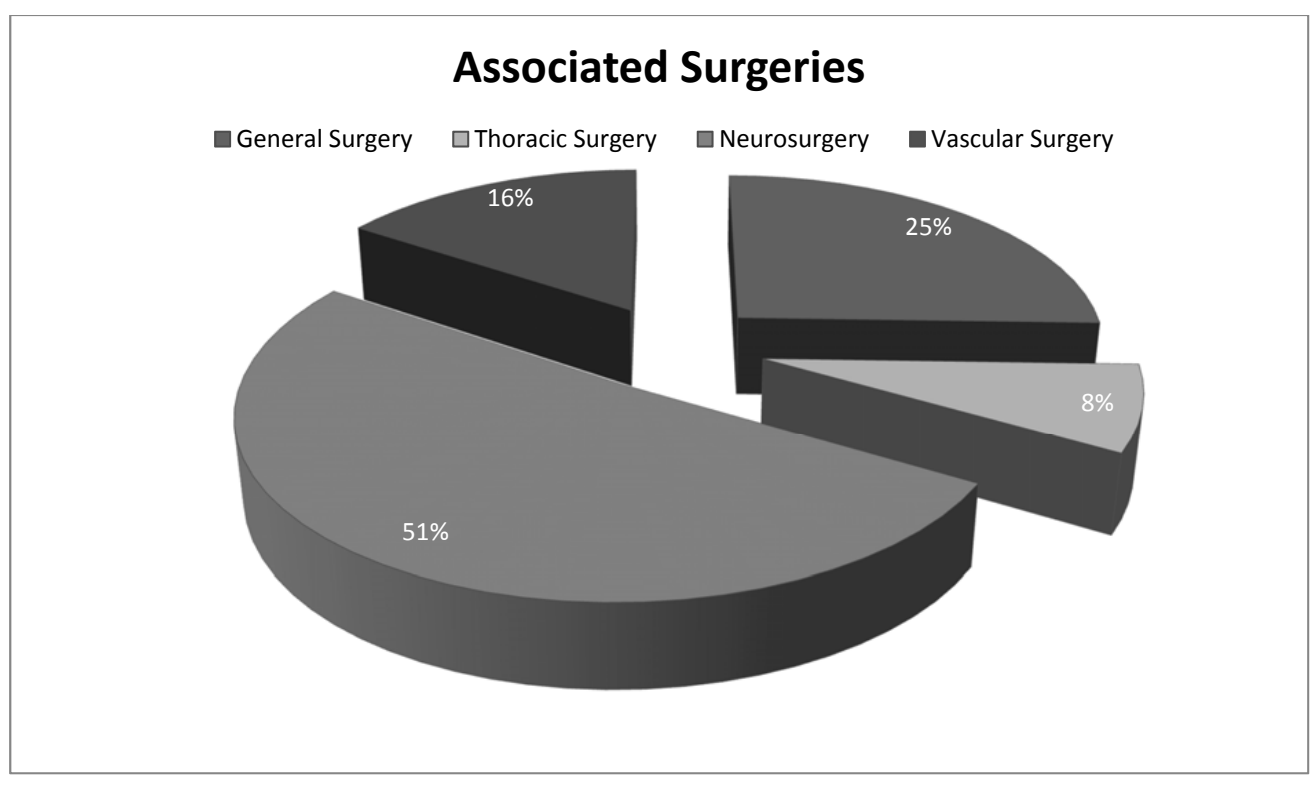

Fig. (3). Distribution of surgical conditions that presented in association with the orthopaedic condition.

was associated with a condition requiring thoracic surgery as along with vascular surgery in $3.85 \%$ of the cases. Two people died in this subgroup: one patient presenting a neurosurgical condition along with the orthopaedic one, and another patient who also presented with a condition that had to be treated by general surgeons, representing $0.96 \%$ of the total.

As for vascular conditions, this condition was present as the only other association with the orthopaedic condition in $2.40 \%$ of the cases, while it was associated with the other analyzed surgeries in one case each. Two people of this subgroup died $(0.96 \%$ of the total cohort), with one patient presenting only the vascular condition and the other presenting a condition that had to be treated by general surgeons.

The study population was also divided into two big subgroups: deceased and weekend traumas.

The deceased group consisted of 17 patients, representing a mortality rate of $8.17 \%$; 13 patients were males and 4 patients were females, with a ratio of 3.25 to 1 (mean age 53.1, min 19, $\max 84$ ). Orthopaedic lesions consisted of a fracture of shin bone and fibula in two cases, a clavicular fracture, a patellar fracture and a pelvic ring fracture, while in the remaining 12 cases, an orthopaedic surgeon was convened to the Emergency Department in the setting of a trauma team and in consideration of the traumatic mechanism which could have implied an orthopaedic condition, but the patient died before such a diagnosis could be made. Only 2 patients $(11.76 \%)$ could be treated within 2 hours from the admittance. One of them underwent limb amputation and the other one underwent external fixation. The other 15 patients $(88.24 \%)$ died before any orthopaedic intervention could be made. Seven patients were admitted on a weekend day $(41.18 \%)$, and 10 on a week day $(58.82 \%)$. Three patients $(17.65 \%)$ had an associated general surgery condition (1 massive intestinal infarction, 1 ruptured spleen, 1 hepatic laceration) and died after emergency surgery; only 2 patients $(11.76 \%)$ also had thoracic trauma (1 hemothorax,
1 lobar rupture). Neurosurgery was not associated with general surgery conditions, while a vascular lesion (1 compartmental syndrome) was present in one case $(5.88 \%)$. Neurological condition was present in 2 cases $(11.76 \%)$ without any other associated lesion than the orthopaedic condition; instead, vascular condition was present as the only other comorbidity in 1 case $(5.88 \%)$.

The weekend trauma group consisted of 88 patients $(42.31 \%)$; 68 were males $(77.27 \%)$ and 20 were females $(22.73 \%)$, with a ratio of 3.4 to 1 (mean age 41.14 , min 14 , max 83). Seven patients died before undergoing orthopaedic surgery, $9(10.23 \%)$ did not have a condition requiring orthopaedic surgery, and $72(81.82 \%)$ underwent a procedure. Out of them, 77.78\% underwent an ETC approach, while $22.22 \%$ had a damage control; 32 patients $(44.44 \%)$ were treated within 6 hours, 7 patients $(9.72 \%)$ between 6 and 12 hours, 2 patients $(2.78 \%)$ were treated between 12 and 24 hours; 4 patients were treated between 24 and 72 hours and 27 patients $(37.50 \%)$ were treated after 72 hours.

Due to the very low number of patients that died after an orthopaedic procedure was indicated, a correlation between mortality and an orthopaedic surgical procedure timing was not found.

Analyzing laboratory data revealed an interesting correlation between mortality and: blood pressure, platelet count, cardiac frequency, hematocrit, hemoglobin and age.

Blood pressure was recorded for 170 of the 208 analyzed patients; as a cut-off point, a value of $90 \mathrm{mmHg}$ was chosen. Fisher's test was applied, and the difference between the two groups appeared statistically significant with a $\mathrm{P}<0.0007$.

A second correlation was made between mortality and platelet count. A total of 187 patients were assessed, and a value of $150,000 \mathrm{PLT} / \mathrm{mL}$ was chosen as the cut-off point. Fisher's test revealed a statistically significant difference. 
Cardiac frequency was correlated to mortality by dividing the patients into 2 groups and considering as cut-off point a value of $90 \mathrm{bpm}$; no statistical difference was noted.

As for the hemoglobin value, all groups of patients were analyzed considering a $10 \mathrm{~g} / \mathrm{dL}$ as the cut-off value. A statistically significant difference was noted. The last laboratory data were hematocrit, which showed a significant difference when choosing a 30\% cut-off value.

The correlation for age showed no significant difference.

A graphical representation is provided by Fig. (4A-E).

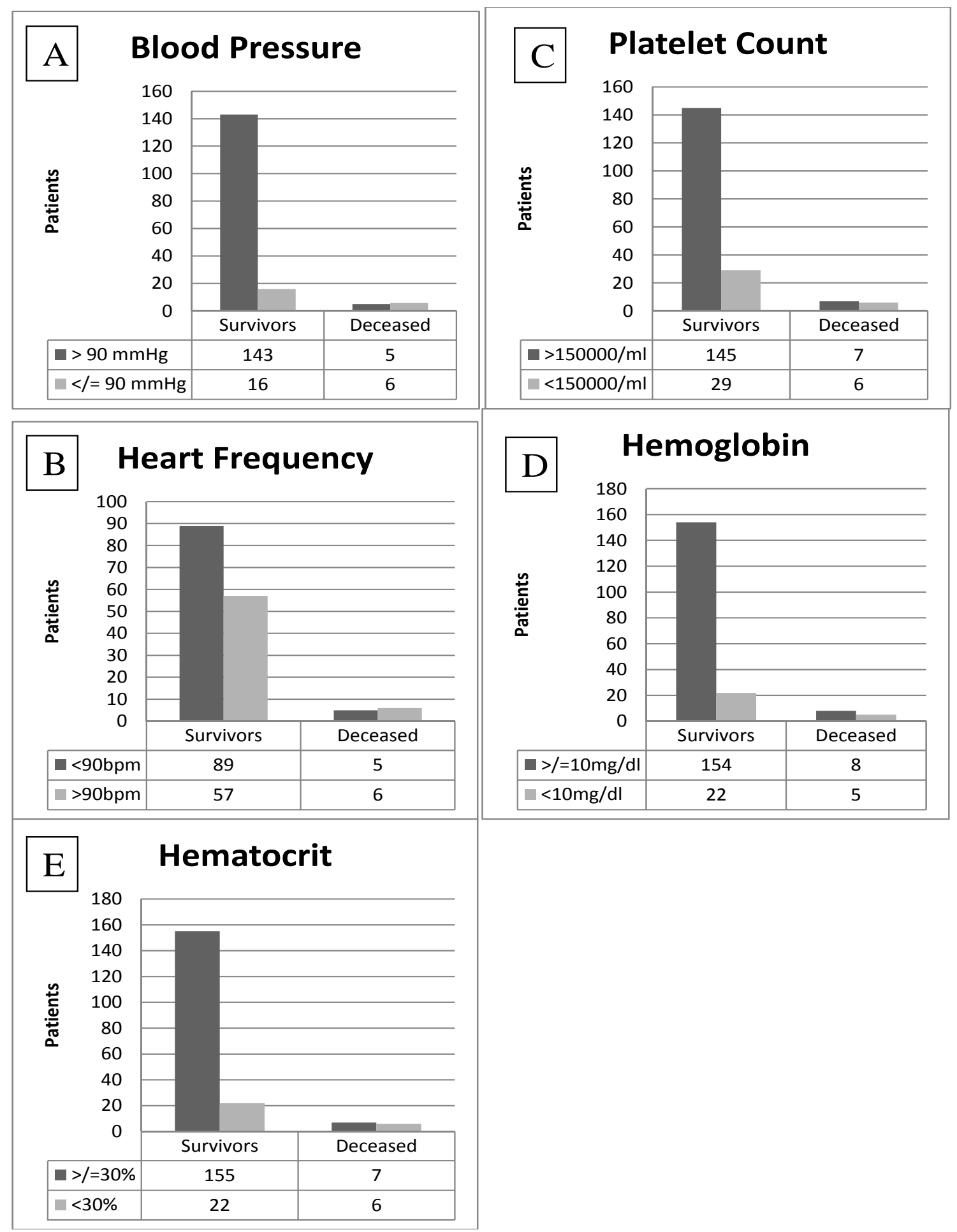

Fig. (4). Histograms of statistically significant laboratory data: A-Blood Pressure; B- Heart frequency; C- Platelet Count; D- Hemoglobin; EHematocrit. Left columns represent survivor groups, right columns represent deceased groups. Cut-offs chosen are based on available literature. 
Glasgow Come Scale and body temperature were not obtained for every patient at the time of hospitalization and therefore not analyzed in this paper.

Lastly, the analysis of deaths which occurred during weekdays and weekends showed no statistical significance being $8.33 \%$ and $7.95 \%$, respectively.

\section{DISCUSSION}

The data from this paper demonstrate that our institution is in line with the recent literature [2, 23, 24], with a $72 \%$ of cases treated before 48 hours.

The great variability of clinical conditions for every patient, as already proved by other authors [24], makes analyses much more complicated and explains why there is still no consensus on the surgical timing debate.

Rixen [24] underlines that even if there is growing evidence on the benefits of an early treatment like damage control orthopaedics, there is no supremacy of this approach in comparison with other methods according to Evidence Based Medicine.

It seems reasonable to think that the surgeon's experience and personal technical skills are the main elements to properly manage a patient who has been a victim of a polytrauma and to obtain a favorable outcome.

In contrast with this paper, Morshed et al. [25] demonstrated an increase in mortality for those patients treated in the first 12 hours if compared to those treated between the $12^{\text {th }}$ and $24^{\text {th }}$ hour after hospitalization and to those treated between the $24^{\text {th }}$ and $48^{\text {th }}$ hour or later.

Correlating mortality with other clinical and laboratory parameters, this paper, as previously described by Matsumoto et al. [21], noted a very strong correlation and statistically significant difference between patients with blood pressure $>90 \mathrm{mmHg}$ and those with blood pressure $<90 \mathrm{mmHg}$ when accepted in the emergency department. It is desirable to obtain at least one blood pressure measurement in traumatic patients and to consider particularly at risk those below the $90 \mathrm{mmHg}$ value. Those patients necessitate hemodynamic stabilization as the first approach and only at a later stage orthopaedic injuries should be taken care of, as previously stated in another paper by Pape et al. [17]. Platelet count is also a matter of concern, and it is related to increased mortality; it should always be taken into account when a patient victim of a polytrauma is admitted to an emergency department.

Hematocrit is another parameter associated with blood loss severity and, therefore, to the clinical gravity of the patient. This paper considered a value of $30 \%$ as cut-off point in the hematocrit; patients with a lower value showed higher and statistically relevant mortality when compared to those with a higher value. The same holds true for hemoglobin when a cut-off value of $10 \mathrm{mg} / \mathrm{dL}$ is taken into consideration, showing a $p=0.023$ statistical significance and representing another parameter that should always be taken into account before taking a surgical decision.

Matsumoto et al. [21] also showed that a body temperature lower than $35.5^{\circ}$ Celsius is of high importance for DCO actuation. This parameter, unfortunately, was not registered in every patient's record reviewed in this paper, and therefore not analyzed; this represents a limitation in the choice of both surgical timing and surgical technique.

We did not find a correlation between week or weekend days and mortality.

\section{CONCLUSION}

According to our findings, orthopaedic surgical timing does not influence the survivorship of a patient who has been a victim of a polytrauma. This is in accordance with the majority of literature. A proper consensus is still far from being reached considering the wide range of contrasting evidence and the clinical importance of this aspect in clinical practice. The 15 deaths recorded in 3 years happened before any orthopaedic treatment could be given which makes it impossible to state that the patients addressed to the orthopaedic surgeon had already been clinically stabilized. As an indirect observation, it could be derived from this paper that orthopaedic surgery procedures should be performed early only in the setting of a hemodynamically unstable patient, if it can contribute to his/her stabilization, and only after other life-saving surgeries have been performed; in any other situation, the patient's orthopaedic treatment can be safely delayed in favor of a one-time orthopaedic surgery if ETC is not applicable at the first stage. Evidence of a higher risk of mortality can suggest the surgeon to delay surgery or to apply the principles of DCO. A statistically significant difference was noted between mortality and some clinical and laboratory parameters obtained at the admittance of the patient in the emergency department; those parameters should be used by the orthopaedic surgeon or by the trauma leader in order to decide when to proceed with orthopaedic surgery.

\section{CONFLICT OF INTEREST}

The authors confirm that this article content has no conflict of interest.

\section{ACKNOWLEDGEMENTS}

The authors would like to thank Mr. David Massey for his revision of the English Language.

\section{REFERENCES}

[1] Pape H, Tonetta P, Tarkin I, Tzioupis C, Sabeson V, Olson S. Timing of fracture fixation in multitrauma patients: the role of early total care and damage control surgery. J Am Acad Orthop Surg 2009; 17(9): 541-9.

[2] Ferguson T, Lee MA. Surgical timing of treating injured extremities: an evolving concept of urgency. Instr Course Lect 2013; 62: 17-28.

[3] Balogh ZJ, Reumann MK, Gruen RL, et al. Advances and future directions for management of trauma patients with musculoskeletal injuries. Lancet 2012 22; 380(9847): 1109-19.

[4] Beck JP, Collins JA. Theoretical and clinical aspect of postraumatic fat embolism syndrome. In: Calandruccio RA, Ed. The American Academy of Ortopaedics Surgeons: Instr Course Lect Mosby St Louis MO 1973; 22: 38-87.

[5] Tscherne H, Schreyer H, Magerl F. Pulmonary and cardiac x-ray findings in traumatic fat embolism. Fortschr Geb Rontgenstr Nuklearmed 1967; 106(5): 703-10. 
[6] Smith JE. The results of early and delayed fixation of fractures of the shaft of the femour. J Bone Joint Surg Br 1974; 56B(3): 469-77.

[7] Hansen ST, Winquist RA. Closed Intramedullary Nailing of the Femur: Kuntscher Technique with Reaming. Clin Orthop Relat Res 1979; 138: 56-61.

[8] Seibel R, LaDuca J, Hassett JM. Blunt multiple trauma, femour traction, and the pulmonary failure tic state. Ann Surg 1985; 202(3): 283-95.

[9] Goris RJ, Gimbrere JS, Van Niekerk JL, Schoots FJ, Booy LH. Early osteosyntesis and prophylactic mechanical ventilation in the multitrauma patient. J Trauma 1982; 22(11): 895-903.

[10] Taeger G, Ruchholtz S, Waydhas C, Lewan U, Schmidt B, NastKolb D. Damage control orthopedics in patients with multiple injuries is effective, time saving, and safe. J Trauma 2005; 59 (2): 408-15.

[11] Johnson KD, Cadambi A, Seibert GB. Incidence of adult respiratory distress syndrome in patients with multiple musculoskeletal injuries: effect of early operative stabilization of fractures. J Trauma-Injury Infection Critical Care 1985; 25(5): 37584.

[12] Border JR. Death from severe thrauma: open fractures to multiple organ disfunction syndrome. J Trauma 1995; 39(1): 12-22.

[13] Giannoudis PV, Abbott C, Stone M, Bellamy MC, Smith RM. Fatal systemic inflammatory response syndrome following early bilateral femoral nailing. Intensive Care Med 1998; 24(6): 641-2.

[14] Scalea TM, Boswell A, Scott JD, Mitchell KA, Kramer ME, Pollak AN. External fixation as a bridge to intramedullary nailing for patients with multiple injuries and with femur fractures: Damage control orthopedics. J Trauma 2000; 48(4): 621-3.

[15] Pape HC, Grimme K, Van Grinsven M, et al. Impact of intramedullary instrumentation versus damage control for femoral fractures on immunoinfiammatory parameters: prospective randomized analysis by the EPOFF Study group. J Trauma 2003; 55(1): 7-13

[16] Rotondo MF, Schwab CW, McGonigal MD, et al. "Damage control": an approach for improved survival in exsanguinating penetrating abdominal injury. J Trauma 1993; 35(3): 375-382.
[17] Pape HC, Rixen D, Morley J. Impact of the method of initial stabilization for femoral shaft fractures in patients with multiple injuries at risk for complications (borderline patients). Ann Surg 2007; 246(3): 491-501.

[18] Morshed S, Miclau T, Bembom O, Cohen M, Knudson MM, Colford JM. Delayed internal fixation of femoral shaft fracture reduces mortality among patients with multisystem trauma. J Bone Joint Surg Am 2009; 91(1): 3-13.

[19] Pape HC, Regel G, Tscherne H. Local and systemic effects of fat embolization after intramedullary reaming and its influence by cofactors. Techniques in Orthopaedics 1996; 11: 2-13.

[20] Rixen D, Steinhausen E, Sauerland S. Protocol for a randomized controlled trial on risk adapted damage control orthopedic surgery of femur shaft fractures in multiple trauma patients. Trials 2009; 10: 72 .

[21] Matsumoto H, Mashiko K, Sakamoto Y, Kutsukata N, Hara Y, Yokota H. A new look at criteria for damage control surgery. J Nippon Med Sch 2010; 77(1): 13-20.

[22] Pape HC, Giannoudis GP, Krettek C. The timing of fracture treatment in polytrauma patients: reverence of damage control orthopaedic surgery. Am J Surg 2002; 183: 622-9.

[23] Nowotarski PJ, Turen Ch, Brumback RJ, Scarboro JM. Conversion of external fixation to intramedullary nailing for fractures of the femur in multiply injured patients. J Bone Joint Surg Am 2000; 82(6): 781-8.

[24] Rixen D, Steinhausen E, Sauerland S, et al. Protocol for a randomized controlled trial on risk adapted damage control orthopaedic surgey of femour shaft fractures in multiple trauma patients. Trials 2009; 10: 72-81.

[25] Morshed S, Miclau T $3^{\text {rd }}$, Bembom O, Cohen M, Knudson MM, Colford M Jr. Delayed internal fixation of femoral shaft fractures reduces mortality among patients with multisystem trauma. J Bone Jt Surg 2009; 91(1): 3-13.

[26] Pape HC, Giannoudis PV, Krettek C, Trentz O. Timing of fixation of major fractures in blunt polytrauma: role of conventional indicators in clinical decision making. J Orthop Trauma 2005; 19(8): 551-62. 